\title{
A novel treatment approach to infected nonunion of long bones without systemic antibiotics
}

\author{
Karim Z. Masrouha ${ }^{1} \cdot$ Michael E. Raad $^{1} \cdot$ Said S. Saghieh ${ }^{1}$
}

Received: 8 July 2016 / Accepted: 14 January 2018 / Published online: 29 January 2018

(c) The Author(s) 2018. This article is an open access publication

\begin{abstract}
Infected nonunion of long bones may require intravenous antibiotics over a lengthy period which may result in a high rate of complications. This study aims to assess the efficacy of local antibiotics used as a replacement to prolonged intravenous therapy. Thirteen patients with infected nonunion of long bones who failed at least one previous surgery were included. The infection was treated through extensive debridement, application of antibiotic-impregnated calcium sulphate pellets and the bone stabilized with external fixation. These patients were monitored for union and infection by clinical signs, laboratory values, and radiographs over a period of 24 months. The results support an eradication of infection and union in all patients with no antibiotic-associated complications. Local antibiotic delivery using calcium sulphate pellets provides an effective method for treatment of nonunion in long bones and is free of the complications from the intravenous route.
\end{abstract}

Keywords Infection $\cdot$ Nonunion $\cdot$ Local antibiotics $\cdot$ Calcium sulphate $\cdot$ Bone pellet $\cdot$ Long bones

\section{Introduction}

Infected nonunion is a dreaded complication of the long bone fractures because its management is challenging to both the patient and the orthopaedic surgeon [1]. Management involves surgical debridement, tissue reconstruction, a long-course of antibiotics and opioids. The complexity of care and its prolonged duration increases the cost of the treatment for the patient [2]. In addition, adverse sequelae may lead to bone loss, residual deformity, or even amputation [1].

Recent advances in surgical technique have tilted the balance towards limb salvage surgery [1]; however, only a few high-quality studies exist which describe the most beneficial and cost-effective post-operative antimicrobial treatment approach [3]. Currently, the most widely used approach is extensive surgical debridement followed by 6 weeks of highdose intravenous (IV) antibiotics [4].

The effective antibiotic penetration and eradication of infection requires 10-100 times the minimum inhibitory

Said S. Saghieh

ss15@aub.edu.lb

1 Department of Surgery, Orthopedics Division, American University of Beirut Medical Center, P. O. Box 11-0236, Riad El-Solh, Beirut 1107 2020, Lebanon concentration (MIC). This high dose is required due to the bacterial biofilm formation and the local milieu at the infection site [5]. When systemic antibiotics are administered for a prolonged period, there is a significant risk of complications. The IV route requires frequent replacement because it is associated with IV-line thrombosis, infection, and stenosis [6]. Other complications include, but are not limited to, haematologic, renal, dermatologic, gastrointestinal, and other metabolic problems such as lactic acidosis [7]. Certain antibiotics used in the treatment of osteomyelitis are considered more toxic than others, particularly aminoglycosides and vancomycin. These two antibiotics require frequent measurement of trough and creatinine levels, further contributing to the burden of treatment [7]. The high frequency of Staphylococcus aureus infection and its high rates of methicillin resistance [8] dictate the use of vancomycin as a first-line treatment. Although effective, vancomycin is associated with nephrotoxicity in as many as $43 \%$ of patients in some studies [9].

The use of a local antibiotic delivery conduit was popularized in Europe during the 1970s [5]. It is associated with lower rates of systemic antibiotic toxicity, allowed for filling of the defect created by extensive debridement, and was considerably cheaper than prolonged courses of systemic antibiotics [8]. Polymethyl-methacrylate (PMMA) is one of the most commonly used antibiotic conduits today but requires 
further surgery for removal and may be associated with bead infection after elution of the antibiotic has completed [10]. Recently, synthetic calcium sulphate has gained popularity as an attractive alternative due to its predictable drug-eluting properties, osteoconductivity, biodegradability and obviating additional surgery for removal [11].

The beneficial effects of this treatment modality necessitate further study to document its efficiency in the absence of systemic antibiotic use. The aim of our study is to evaluate the resolution of infection and bone healing in patients with infected nonunion of long bones treated with extensive debridement, bone reconstruction and antibiotic-impregnated calcium sulphate pellets without subsequent use of systemic antibiotics.

\section{Materials and methods}

This is a retrospective cohort study of 13 consecutive patients with infected nonunion of long bone from two affiliated medical centres between 2003 and 2012. Institutional review board approval was obtained from both institutions prior to initiation of the study. All patients included in the study had stage IV osteomyelitis as per the Cierney-Mader classification system [12], failed at least one previous surgery, and were operated on by one surgeon. All patients were followed up post-operatively for a period of 24 months before being discharged from the clinic. Patients under the age of 18 and with infected nonunions other that long bones were excluded.

\section{Surgical procedure}

Use of the bone graft substitute was an integral part of our protocol. Any previously implanted hardware was removed. Thorough debridement of all nonviable bone and necrotic soft tissue was performed. The site of infection was irrigated thoroughly with large quantities of Normal Saline. All surgically extracted tissues were sent for culture. The paprika sign was used to detect viable bone. An external fixator was then applied to hold the nonunion site. When needed, a remote osteotomy was performed for gradual distraction. Bone regeneration was planned dependent upon the bone loss and according to the principles of histogenesis [13]. Then, selfprepared calcium sulphate pellets were used to fill the defect (Stimulan Kit, Biocomposites Ltd., Staffordshire, England).

The paste was prepared according to the instructions in the supplied kit and mixed with $1 \mathrm{~g}$ of vancomycin and $240 \mathrm{mg}$ of gentamicin. The result was a combination of pellets impregnated with antibiotics, measuring $3 \mathrm{~mm}$ and $4.8 \mathrm{~mm}$ in diameter.

\section{Outcomes measured}

The study outcomes were union and the presence of infection. Bone union was defined as bridging of three out of four cortices on two orthogonal radiographs [14]. Infection was assessed by clinical signs (erythema, drainage, and wound problems) and further need for systemic antibiotics. These parameters were assessed during outpatient follow-up visits as part of the standard of care and biannually after discharge from the clinic.

Also, the erythrocyte sedimentation rate (ESR) was measured, as per the hospital's protocol, and reported for each patient post-operatively. Normal ESR ranges between 0 and $15 \mathrm{~mm} / \mathrm{h}$ for those younger than 50 and between 1 and $20 \mathrm{~mm} / \mathrm{h}$ for those older than 50 . Microbiological assays from specimens that were extracted intraoperatively were analysed. The mean and standard deviation (SD) were obtained for continuous data sets, and others were reported as modes.

\section{Results}

\section{Patient characteristics}

The average age was 35 years (18-63 years). All patients were male. The majority of structural defects involved the tibia; the femur and humerus were a lower proportion (Table 1). All patients had previously been operated on with an average of three surgeries (Table 1). Pre-operatively, nine patients had draining sinuses and four had evidence of skin necrosis with soft tissue defects (Table 1).

Eight patients underwent acute shortening with pellets implanted at the time of surgery. Five had bone transport with pellets implanted at the time of docking site grafting. All patients had the external fixator in place until bony union. One patient had to undergo bone grafting for nonunion at the docking site, which subsequently healed.

\section{Union}

All patients achieved union during the follow-up period as evidenced on radiographs (Fig. 1). The average time to union was 5.5 months (3-11 months) (Table 2).

\section{Infection}

The ESR was found to be normal in all 13 patients postoperatively with an average of $9.8 \pm 3.5 \mathrm{~mm} / \mathrm{h}$ (Table 2). All cases involving a draining sinus, or with local signs of 
Table 1 Patient demographics and pre-operative characteristics

\begin{tabular}{llllll}
\hline Patient & Age & Sex & Fracture site & $\begin{array}{l}\text { Number of previous } \\
\text { surgeries }\end{array}$ & Signs of infection \\
\hline 1 & 35 & Male & Tibia & 3 & Soft tissue defect \\
2 & 18 & Male & Femur & 3 & Draining sinus \\
3 & 50 & Male & Tibia & 9 & Soft tissue defect \\
4 & 44 & Male & Humerus & 3 & Draining sinus \\
5 & 28 & Male & Tibia & 5 & Draining sinus \\
6 & 24 & Male & Femur & 2 & Draining sinus \\
7 & 22 & Male & Tibia & 4 & Draining sinus \\
8 & 25 & Male & Tibia & 3 & Soft tissue defect \\
9 & 22 & Male & Tibia & 1 & Draining sinus \\
10 & 43 & Male & Humerus & 1 & Draining sinus \\
11 & 45 & Male & Tibia & 2 & Draining sinus \\
12 & 63 & Male & Tibia & 3 & Draining sinus \\
13 & 40 & Male & Tibia & 4 & Soft tissue defect \\
Average & $35.3 \pm 13.5$ & Male & Tibia & 3 & Draining sinus \\
\hline
\end{tabular}

All averages indicate mode except age whose average is expressed as mean \pm standard deviation infection such as erythema, warmth, swelling or pain over the treated segment resolved in the post-operative follow-up period. No recurrences were noted in that time (Table 2).

\section{Microbiology}

Bone cultures were taken from all patients intraoperatively. Microbiological analyses showed coagulase-negative Staphylococcus (5 cases) to be the most common. The other culture results were as follows: Staphylococcus aureus (2 cases), Escherichia Coli (2 cases), Enterobacter cloacae (1 case), and three cases had mixed infections involving gramnegative bacilli and gram-positive cocci (Table 2).

\section{Need for systemic antimicrobial therapy after discharge}

Patients received IV antibiotics immediately after debridement and during their hospital stay, which was typically three to 5 days. None of the patients received systemic antibiotics after being discharged from the hospital.

\section{Discussion}

Our treatment modality eradicated infection and allowed for bone healing, while sparing patients prolonged courses of IV antibiotics. All of our patients achieved union after an average of 5.5 months and had no clinical signs of infection within the period of follow-up. None of the patients required IV antibiotics after leaving the hospital and the only antibiotic to which they were exposed was that impregnated in the calcium sulphate beads.
Local antibiotic beads allow the delivery of high antibiotic concentrations locally with very low levels in the systemic circulation [15]. Calcium sulphate offers the advantage of biodegradability over PMMA, sparing patients a second surgery for removal. Its osteoinductive properties also aided bone healing and filling the bone gap created by extensive debridement [16].

Our choice of surgical procedure was based on our own experience and a review by Peter et al. [17], in which 34 clinical trials for the treatment of infected nonunion of the long bones were studied. Although there is no consensus, the highest cure rates are observed with the two-stage procedure of debridement, antibiotic beads, and planned secondary fixations [17]. We believe that the surgical procedure is an integral part of the treatment protocol, and we emphasize the role of thorough debridement of any necrotic tissue before the application of beads. Selhi et al. [18] looked at a treatment protocol similar to ours but with PMMA beads applied to 16 patients. Thirteen per cent of the patients in their study failed to achieve union and eradication of infection. This was attributed to inadequate debridement and the choice of bone cement [18]. Calcium sulphate has been shown to be just as good or even superior to PMMA as a carrier of antibiotics to infected surgical sites [19], and our study supports the efficiency of calcium sulphate.

Another large series by Ferguson et al. looked at 195 patients treated with surgery and calcium sulphate pellets, followed by intravenous antibiotics for 6 weeks. After the initial treatment, $91.8 \%$ of patients achieved eradication of infection [20]. Higher rates of success were achieved in our study and without extended use of intravenous antibiotics. However, the average age in their study was 46.1 years (16.1-82 years), 11 years older than the average age in 

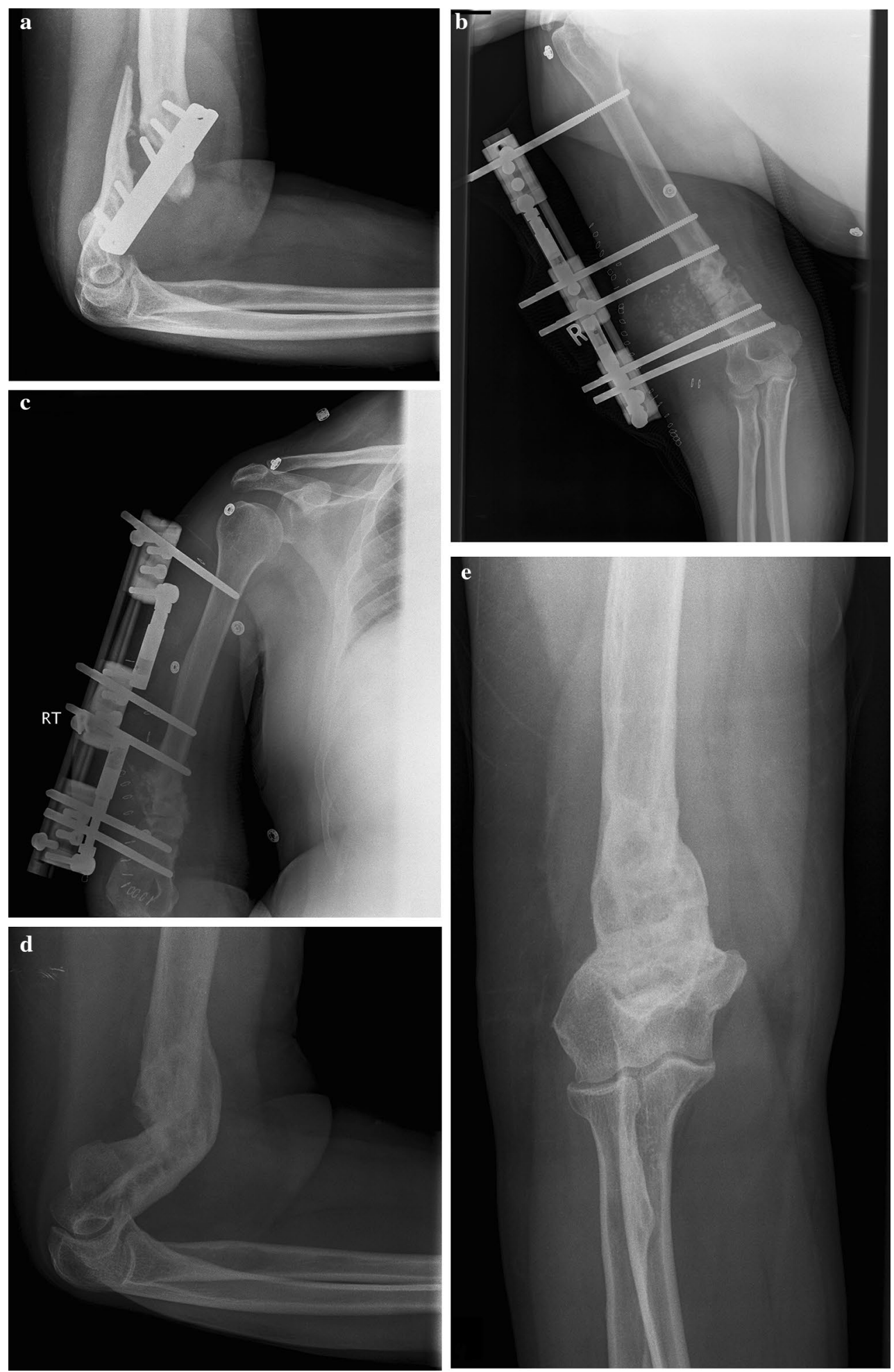

Fig. 1 a Pre-operative radiograph of Patient 10 shows nonunion at the place; $\mathbf{d}$ and $\mathbf{e}$ follow-up anteroposterior and lateral radiograph shows distal end of the humerus; $\mathbf{b}$ and $\mathbf{c}$ immediate post-operative radiothe healed fracture 
Table 2 Post-operative union and infection parameter outcomes

\begin{tabular}{|c|c|c|c|c|c|}
\hline Patient & $\begin{array}{l}\text { Time to union } \\
\text { (months) }\end{array}$ & $\begin{array}{l}\text { ESR post-operatively } \\
(\mathrm{mm} / \mathrm{h})\end{array}$ & Culture results & $\begin{array}{l}\text { Resolution of clini- } \\
\text { cal signs }\end{array}$ & $\begin{array}{l}\text { Outpatient sys- } \\
\text { temic antibiotic } \\
\text { therapy }\end{array}$ \\
\hline 1 & 3 & 9 & Mixed & Yes & None \\
\hline 2 & 11 & 11 & Mixed & Yes & None \\
\hline 3 & 3 & 15 & Escherichia.coli $($ ESBL) & Yes & None \\
\hline 4 & 4 & 5 & Staphylococcus coagulase negative & Yes & None \\
\hline 5 & 4 & 7 & E. coli & Yes & None \\
\hline 6 & 8 & 11 & Staphylococcus aureus & Yes & None \\
\hline 7 & 11 & 4 & S. aureus & Yes & None \\
\hline 8 & 6 & 8 & Staphylococcus coagulase negative & Yes & None \\
\hline 9 & 3 & 13 & Mixed & Yes & None \\
\hline 10 & 6 & 11 & Staphylococcus coagulase negative & Yes & None \\
\hline 11 & 4 & 7 & Staphylococcus coagulase Negative & Yes & None \\
\hline 12 & 6 & 12 & Eneterobacter cloacae & Yes & None \\
\hline 13 & 3 & 15 & Staphylococcus coagulase negative & Yes & None \\
\hline Average & $5.5 \pm 2.9$ & $9.8 \pm 3.5$ & Staphylococcus coagulase negative & Yes & None \\
\hline
\end{tabular}

All averages indicate mode except time to union and erythrocyte sedimentation rate (ESR) whose averages are expressed as mean \pm standard deviation. The mixed culture result included more than one of the following Pseudomonas cepaciae; Klebsiella oxitocae; Pseudomonas aeroginosa; Staphylococcus aureus; and/or coagulase-negative Staphylococcus

our study sample (35.3 years), and our oldest patient was 63 years old. Advancing age has a well-known impact on the regenerative capacity of bone [21] which might have influenced the success rate.

Gauland et al. [11] reported eradication of infection in $86.4 \%$ of 337 subjects treated with the same modality as ours. The lower rate might be partly explained by the difference in our inclusion criteria, which included patients with osteomyelitis of the lower extremity only. The foot, being a distant organ, is associated with poorer perfusion when compared to the long bones and much infective pathology in this area associated with diabetes which tips the balance in favour of continuing infection [22]. It is worth mentioning that the vancomycin dose mixed with the $10 \mathrm{~mL}$ calcium sulphate paste is higher in our series (1 g versus $500 \mathrm{mg}$ ). However, there is insufficient data in the literature regarding the effect of the antibiotic dose on treatment outcome for this treatment modality.

There is growing evidence for the idea that long-term intravenous antibiotics in infected nonunion does not guarantee a cure and is associated with high levels of antibiotic and catheter-related complications [23]. As such, the authors attribute the success of treatment to the aggressive debridement as well as the elution and bone healing properties of calcium sulphate bone substitute.

We acknowledge the limitations inherent in all retrospective cohorts. Although this study had a small sample size, it adds to the body of evidence of antibiotic-impregnated bone substitute treatment of patients with infected nonunion. We recommend further investigations through larger series and randomized control trials comparing it to different modalities of treatment. Although most recurrences of osteomyelitis occur within 2 years of treatment [24], it is well known to recur up to several decades later [22]. Therefore, a longer follow-up period will also allow for better evaluation of treatment outcomes.

\section{Conclusion}

Based on our study, the use of antibiotic-impregnated calcium sulphate pellets without subsequent extended use of systemic antibiotics holds promise for treatment of infected nonunions of long bones. It offers the advantage of sparing patients the complications and costs of prolonged intravenous antibiotics and is associated with satisfactory outcomes.

\section{Compliance with Ethical Standards}

Conflict of interest The authors declare that they have no conflict of interest.

Ethical approval All procedures performed in studies involving human participants were in accordance with the ethical standards of the institutional research committee, institutional review board (IRB), of both institutions and with the 1964 Declaration of Helsinki and its later amendments or comparable ethical standards.

Informed consent Informed consent was obtained from all individual participants included in the study. 
Open Access This article is distributed under the terms of the Creative Commons Attribution 4.0 International License (http://creativeco mmons.org/licenses/by/4.0/), which permits unrestricted use, distribution, and reproduction in any medium, provided you give appropriate credit to the original author(s) and the source, provide a link to the Creative Commons license, and indicate if changes were made.

\section{References}

1. Bose D, Kugan R, Stubbs D, McNally M (2015) Management of infected nonunion of the long bones by a multidisciplinary team. Bone Joint J 97B(6):814-817. https://doi. org/10.1302/0301-620X.97B6.33276

2. Antonova E, Le TK, Burge R, Mershon J (2013) Tibia shaft fractures: costly burden of nonunions. BMC Musculoskelet Disord 14:42. https://doi.org/10.1186/1471-2474-14-42

3. Fraimow HS (2009) Systemic antimicrobial therapy in osteomyelitis. Semin Plast Surg 23(2):90-99. https://doi. org/10.1055/s-0029-1214161

4. Lazzarini L, Lipsky BA, Mader JT (2005) Antibiotic treatment of osteomyelitis: what have we learned from 30 years of clinical trials? Int J Infect Dis 9(3):127-138. https://doi.org/10.1016/j. ijid.2004.09.009

5. Gogia JS, Meehan JP, Di Cesare PE, Jamali AA (2009) Local antibiotic therapy in osteomyelitis. Semin Plast Surg 23(2):100-107. https://doi.org/10.1055/s-0029-1214162

6. Cheung E, Baerlocher MO, Asch M, Myers A (2009) Venous access: a practical review for 2009. Can Fam Physician 55(5):494-496

7. Marculescu CE, Berbari EF, Cantey JR, Osmon DR (2012) Practical considerations in the use of outpatient antimicrobial therapy for musculoskeletal infections. Mayo Clin Proc 87(1):98-105. https://doi.org/10.1016/j.mayocp.2011.11.005

8. Paladino JA, Poretz D (2010) Outpatient parenteral antimicrobial therapy today. Clin Infect Dis 51(S2):S198-208. https://doi. org/10.1086/653520

9. Van Hal SJ, Paterson DL, Lodise TP (2013) Systematic review and meta-analysis of vancomycin-induced nephrotoxicity associated with dosing schedules that maintain troughs between 15 and 20 milligrams per liter. Antimicrob Agents Chemother 57(2):734 744. https://doi.org/10.1128/AAC.01568-12

10. Neut D, van de Belt H, Stokroos I, van Horn JR, van der Mei HC, Busscher HJ (2001) Biomaterial-associated infection of gentamicin-loaded PMMA beads in orthopaedic revision surgery. J Antimicrob Chemother 47(6):885-891. https://doi.org/10.1093/ $\mathrm{jac} / 47.6 .885$

11. Gauland C (2011) Managing lower-extremity osteomyelitis locally with surgical debridement and synthetic calcium sulphate antibiotic tablets. Adv Skin Wound Care 24(11):515-523. https ://doi.org/10.1097/01.ASW.0000407647.12832.6c

12. Mader JT, Shirtliff M, Calhoun JH (1997) Staging and staging application in osteomyelitis. Clin Infect Dis 25(6):1303-1309

13. Ilizarov GA, Ledyaev VI (1992) The replacement of long tubular bone defects by lengthening distraction osteotomy of one of the fragments. 1969. Clin Orthop Relat Res 280:7-10

14. Whelan DB, Bhandari M, Stephen D, Kreder H, McKee MD, Zdero R, Schemitsch EH (2010) Development of the radiographic union score for tibial fractures for the assessment of tibial fracture healing after intramedullary fixation. J Trauma 68(3):629-632. https://doi.org/10.1097/TA.0b013e3181a7c16d

15. Smilack JD, Flittie WH, Williams TW Jr (1976) Bone concentrations of antimicrobial agents after parenteral administration. Antimicrob Agents Chemother 9(1):169-171

16. Gitelis S, Brebach GT (2002) The treatment of chronic osteomyelitis with a biodegradable antibiotic-impregnated implant. J Orthop Surg (Hong Kong) 10(1):53-60

17. Struijs PA, Poolman RW, Bhandari M (2007) Infected nonunion of the long bones. J Orthop Trauma 21(7):507-511. https://doi. org/10.1097/BOT.0b013e31812e5578

18. Selhi HS, Mahindra P, Yamin M, Jain D, De Long WG, Jr Singh $\mathbf{J}$ (2012) Outcome in patients with an infected nonunion of the long bones treated with a reinforced antibiotic bone cement rod. J Orthop Trauma 26(3):184-188. https://doi.org/10.1097/ BOT.0b013e318225f77c

19. McConoughey SJ, Howlin RP, Wiseman J, Stoodley P, Calhoun JH (2015) Comparing PMMA and calcium sulphate as carriers for the local delivery of antibiotics to infected surgical sites. J Biomed Mater Res B Appl Biomater 103(4):870-877. https://doi. org/10.1002/jbm.b.33247

20. Ferguson JY, Dudareva M, Riley ND, Stubbs D, Atkins BL, McNally MA (2014) The use of a biodegradable antibiotic-loaded calcium sulphate carrier containing tobramycin for the treatment of chronic osteomyelitis: a series of 195 cases. Bone Joint J 96-B(6):829-836. https://doi.org/10.1302/0301-620x.96b6.32756

21. Gruber R, Koch H, Doll BA, Tegtmeier F, Einhorn TA, Hollinger JO (2006) Fracture healing in the elderly patient. Exp Gerontol 41(11):1080-1093. https://doi.org/10.1016/j.exger.2006.09.008

22. Ciampolini J, Harding KG, Pathophysiology of chronic bacterial osteomyelitis (2000) Why do antibiotics fail so often? Postgrad Med J 76(898):479-483. https://doi.org/10.1136/pmj.76.898.479

23. Ceroni D, Regusci M, Pazos JM, Saunders CT, Kaelin A (2003) Risks and complications of prolonged parenteral antibiotic treatment in children with acute osteoarticular infections. Acta Orthop Belg 69(5):400-404

24. Cierny G 3rd, DiPasquale D (2006) Treatment of chronic infection. J Am Acad Orthop Surg 14(10):S105-110. https://doi. org/10.5435/00124635-200600001-00025 